\title{
THE JOURNAL of the AMERICAN OSTEOPATHIC AsSOCIATION
}

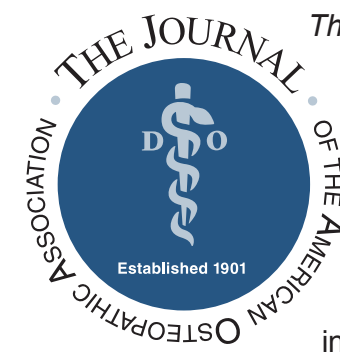

The Journal of the American Osteopathic Association

(JAOA) encourages osteopathic physicians, faculty members and students at colleges of osteopathic medicine, and others within the health care professions to submit comments related to articles published in the JAOA and the mission of the osteopathic medical profession. The JAOA's editors are particularly interested in letters that discuss recently published original research.

Letters must be submitted online at http://www.osteopathic.org/JAOAsubmit. Letters to the editor are considered for publication in the JAOA with the understanding that they have not been published elsewhere and are not simultaneously under consideration by any other publication. All accepted letters to the editor are subject to editing and abridgment.

Although the JAOA welcomes letters to the editor, these contributions have a lower publication priority than other submissions. As a consequence, letters are published only when space allows.

\section{Leg Length Discrepancy and Osteoarthritic Knee Pain in the Elderly}

\section{To the Editor:}

The article by Donald R. Noll, DO, in the September 2013 issue of The Journal of the American Osteopathic Association $(J A O A)^{1}$ was an interesting read despite its small database. It addresses 3 questions regarding postural asymmetry:

1. How do we, the readers of the JAOA, define a short leg?

2. Does a short leg correlate with unilateral knee pain?

3. Can we bypass classical standing lumbosacral (SLS) radiographic evaluation of leg length discrepancy (LLD) by use of visual or palpatory physical findings that would allow us to separate functional treatable causes of LLD in a clinically

efficacious way?

There is congruency in the osteopathic, orthopedic, chiropractic, and physical therapy literature that the reference standard for measurement of anatomic LLD is SLS radiography. Protocols to optimize the comparison of the standing height of the femoral heads ( $\Delta$ FHU [femoral head unleveling], where $\Delta$ indicates a relative quantitative difference on the low or short side $^{2}$ ) and reduce left-right magnification differences date back to $\mathrm{Schwab}^{3}$ in the 1920 s. These protocols were formalized by Denslow et $\mathrm{al}^{4}$ in the osteopathic literature more than 60 years ago. A unilateral dropped foot arch, severe unilateral hip, or knee arthritis would be considered part of the anatomic LLD, even if such conditions developed over time from functional asymmetry.
Anterior or posterior (A-P) rotations of the innominate bones, sacral torsions or shears, tight psoas muscles, or tight quadratus lumborum muscles may affect the apparent LLD in the supine, non-weightbearing position but do not affect the height of the femoral heads in the standing, weight-bearing position.

It is problematic to define short leg as the leg presenting with superior (cephalic) medial malleolus in the supine position, when apparent short leg is actually more accurate, including functional and anatomic components.

Thirty years ago, Travell and Simons ${ }^{5}$ reviewed a number of studies looking at anatomic LLD in symptomatic and asymptomatic populations. If we assume a similar prevalence of anatomic LLD, then the challenge is to show that some combination of physical findings can identify the functional causes of LLD and that osteopathic manipulative treatment can resolve or stabilize symptoms of unilateral knee pain before obtaining standing lumbar radiographs and managing anatomic LLD with a heel or foot lift.

Irvin ${ }^{6}$ showed more than 20 years ago that patients with chronic low back pain and sacral base unleveling $(\triangle \mathrm{SBU})$ and scoliosis convex to the ipsilateral side (the most common compensatory pattern) would have reduced pain and decreased scoliotic curve with leveling of the sacral base by use of a heel lift.

It is difficult to argue that apparent functional factors play a major role in LLD but dismiss $\triangle \mathrm{SBU}$, sacral torsion, and sacral shear as "not predominant functional factors influencing LLD." This interpretation came about by 
assuming that $\Delta \mathrm{SBU}$ will result in uneven iliac crests in the supine position.

On standing A-P view of the SLS radiograph, we typically measure the height of the iliac crest on the posterior aspect along the attachment of the quadrate muscle. A left-to-right difference reflects primarily rotation of the innominate bone around a horizontal axis in the sagittal plane but may also reflect an anatomic short leg by relative rotation of the pelvis about an A-P axis in the coronal plane.

With the patient in the standing position, we tend to evaluate iliac crest heights on the lateral aspects, where a difference reflects more of an anatomic LLD. In the supine, non-weight-bearing position, iliac crest height in the coronal plane is more likely to be affected by unilateral spasm or contracture of quadratus/psoas/erector spinae muscle groups in the absence of gravitational loading, exposing more functional causes for LLD and iliac crest height asymmetry.

In a 2004 article in which 421 cases were reviewed, ${ }^{2}$ iliac crest height in SLS radiographs correlated strongly with $\Delta$ FHU. Historically, the frequency of $\Delta F H U$ for patients with low back pain is higher than that of control patients for cutoffs of 4-, 10-, and 15-mm levels. The type I pattern, in which $\Delta \mathrm{FHU}$ and $\Delta \mathrm{SBU}$ are parallel, is the most common compensatory response to a short leg, but as the $\Delta$ FHU cutoff increases, the added $\Delta$ SBU of type IB overwhelms the prevalence of pure $\triangle F H U$. As the cutoff for $\Delta$ LLD increases, the frequency of $\Delta \mathrm{SBU}$ increases with functional scoliosis tending to be convex to the $\Delta \mathrm{SBU}$.
Dott et $\mathrm{al}^{7}$ found poor correlation of the iliac crest with $\Delta \mathrm{SBU}$, presumably because of functional compensatory mechanisms. The sacrum, which acts as a gear box between the bipedal mechanics of the lower half of the body and the unimodal torso, apparently does not follow the gravitational effect of the $\Delta \mathrm{FHU}$ with the same regularity as the iliac crest.

According to my personal conversations with Phillip Greenman, DO, it was his opinion that $\Delta \mathrm{SBU}$ is more important than $\Delta$ FHU. Whether $\Delta$ SBU is considered anatomic or functional probably does not lessen its importance on spinal mechanics going cephalad or hip or knee mechanics going caudad.

One core question is whether physical examination can distinguish an anatomic short leg from $\triangle \mathrm{SBU}$. The standing and seated forward flexion tests and the relative position of inferior lateral angles in the prone position may be useful in this regard, but we cannot know for certain without correlating those physical measurements to SLS radiographic results in a substantial number of cases. We can assume that $\triangle \mathrm{SBU}$ on standing SLS radiograph, as a result of sacral down sheer or sacral torsion, is a functional LLD, but we also do not have before and after radiologic evidence that classical osteopathic manipulative treatment will reduce or remove $\Delta$ SBU.

My unpublished research (recently rejected for publication by the $J A O A$ ) confirms and supports classical osteopathic theory that the ipsilateral innominate bone tends to rotate anteriorly on the anatomic short leg side as a compensation for the short leg. My future investigations will attempt to correlate the frontal plane pelvic postural asymmetry patterns with compensatory patterns of somatic dysfunction and visual or palpatory findings on physical examination. An algorithm for the differential diagnosis of apparent short leg is available on http://www.drjuhl.com under "Osteopathy."

The greater the number of people thinking about these issues, the better. Osteopathy has always been about science-and intuition. A greater unification theory of somatic dysfunction continues to emerge. (doi:10.7556/jaoa.2014.018)

\section{John H. Juhl, DO}

Adjunct Clinical Professor, Touro College of Osteopathic Medicine in New York City, New York

\section{References}

1. Noll DR. Leg length discrepancy and osteoarthritic knee pain in the elderly: an observational study. J Am Osteopath Assoc. 2013;113(9):670-678.

2. Juhl JH, Ippolito Cremin TM, Russell G. Prevalence of frontal plane pelvic postural asymmetry-part I. J Am Osteopath Assoc. 2004;104(10):411-421.

3. Schwab WA. Principles of manipulative treatment: the low back problem, part III. J Am Osteopath Assoc. 1932;31:253-261.

4. Denslow JS, Chace JA, Gutensohn OR, Kumm MG. Methods in taking and interpreting weight-bearing $\mathrm{x}$-ray films. J Am Osteopath Assoc. 1955;54:663-670.

5. Travell JG, Simons DG. Myofascial Pain and Dysfunction: The Trigger Point Manual, The Lower Extremities. Vol 2. Baltimore, MD: Williams \& Wilkins; 1983.

6. Irvin RE. Reduction of lumbar scoliosis by use of a heel lift to level the sacral base. J Am Osteopath Assoc. 1991;91(1):34,37-44.

7. Dott GA, Hart CL, McKay C. Predictability of sacral base levelness based on iliac crest measurements. J Am Osteopath Assoc. 1994;94(5):383-390. 


\section{Response}

I thank Dr Juhl for his thoughtful letter to the editor ${ }^{1}$ regarding my study published in the September 2013 issue of The Journal of the American Osteopathic Association $(J A O A){ }^{2}$ It was a pleasure to read Dr Juhl's literature review and discussion of pelvic functional mechanics. I will only offer a few additional thoughts for discussion.

Although the standing lumbosacral (SLS) radiograph may be our reference standard, sadly it is not the method of choice for the larger biomedical research community. In 2002, Gurney ${ }^{3}$ reviewed the literature on LLD, and in 2008, Sabharwal and Kumar ${ }^{4}$ published a systemic review of 42 articles describing methods for measuring leg length discrepancy (LLD). Neither of these reviews mentioned the SLS radiographic method of assessing LLD. These researchers do not seem to know about measuring LLD by comparing the relative positions of the heads of the femurs on a standing radiograph. Typically, the rest of the world assesses LLD by measuring the length of each limb by various ways and then comparing the difference between the 2 limbs. Some of these methods are supine and some are standing. There is an indirect method to assess LLD by putting blocks of known height under the apparent short limb until both iliac crests appear level by palpation. ${ }^{5}$

We may forgive these allopathic authors for not knowing about the SLS radiographic method or even Dr Juhl's work for several reasons. First, the literature cited by Dr Juhl in his letter ${ }^{1}$ is rather old, and few researchers do literature searches as far back as the 1950s. Second, the few relatively recent articles describing the SLS radiographic technique ${ }^{6,7}$ do not use the terms limb or leg length in the title, so they are easy to miss. But I think the main reason the SLS radiographic technique was overlooked is that the osteopathic medical profession publishes too few scholarly papers. Collectively, we are failing to make the impact we should have on the larger biomedical research community. The osteopathic medical profession has a wealth of pragmatic knowledge, but it is failing to translate that knowledge into evidence-based, peer-reviewed literature, which is the driver of modern clinical practice. In this sense, Dr Juhl is a hero because he has already done far more than most to counter this problem.

The genesis for my research was a simple clinical observation: when my patients present with unilateral knee pain, more times than not it is on the side of the apparent short leg as measured by the supine medial malleolus physical examination technique. Any osteopathic physician has the skill to check this observation. When I read a study showing that LLD by whole limb length radiographic assessment was associated with progressive and symptomatic knee osteoarthritis in the short limb, ${ }^{8}$ I decided to collect data to see if my observation was so. Assessment by physical examination is far less expensive and easier than radiographic testing and should correlate reasonably well. I opted for an observational study design, one that would allow me to collect the data during my busy office practice. Whereas randomized controlled trials are always better, these projects take considerable time and money to conduct. Confirmatory clinical trials can come later; it is better to start somewhere than nowhere.

I agree completely with Dr Juhl ${ }^{1}$ that this physical examination technique is not the reference standard and should be termed "apparent" LLD. Measuring the supine medial malleolus bony landmarks for leg length is like measuring the length of 2 poles by comparing their relative positions at 1 end without being sure that the other ends are lined up evenly. The beauty of the SLS radiography method is knowing that 1 end is level (the floor). The examiner only needs to measure the relative difference between the femoral head heights to find the anatomic LLD.

I agree that we should not dismiss the role of sacral base unleveling in LLD, especially in the standing position. Nevertheless, I am not sure the sacrum influences apparent LLD when the patient is supine and the iliac crests are approximately equal. Because the femoral bones attach directly to the innominate bones (of which the iliac crests are a part), it seems to me the positions of the innominate bones would govern supine apparent LLD, regardless of sacral base unleveling. I do believe the position of the sacral base is very important, especially for things such as gait, back pain, and scoliosis.

I hope Dr Juhl persists in his efforts to publish the rest of his work in a peerreviewed journal. His work is very important. Reviewers can be overly restrictive, demanding, and even unreasonable, but they have the responsibility of ensuring scientific quality. My study ${ }^{2}$ was rejected by 2 journals and had to be revised multiple 
times before the JAOA would publish it. One journal loved the concept but rejected the manuscript because it was not a randomized controlled clinical trial. The other journal was reluctant to publish it because the data collection was not fully blinded. These are legitimate concerns, but we should be realistic and acknowledge that it takes substantial financial resources to overcome these limitations.

As a profession, we do not invest much money into osteopathic medical research, even though it is the single most important thing we could do to secure our collective future. While the profession is exploding in size, practically doubling since 2000, the spending by the American Osteopathic Association (AOA) on osteopathic research has remained flat. Degenhardt and Standley ${ }^{9}$ recently pointed out that the AOA, through its Council on Research, spends approximately $\$ 250,000$ per year on research from funds originally donated by members of the profession in the 1990s. As of May 31, 2012, there were an estimated 69,429 osteopathic physicians in active practice, ${ }^{10}$ making this amount equivalent to spending approximately $\$ 3.60$ per practicing osteopathic physician per year on research. Or, if the money was divided equally among all of the 29 colleges of osteopathic medicine (not counting branch campuses or additional locations), ${ }^{11}$ each would receive $\$ 8620$ per year to conduct research. This amount is not much money.

It is sad that we encourage osteopathic medical students and residents to conduct small research projects and present posters at research conferences when the profession is failing to invest sufficient funds to support them if they want to go further. Of course, student and resident research projects are of great educational value. Those who complete an original research project from conception to publication have a much deeper understanding of evidencebased medicine. But at current funding levels, virtually none of these students or residents will have an opportunity to develop a career in osteopathic research.

To reach a critical mass, we need to provide sufficient funds to support both developing and established full-time career osteopathic researchers. It is a mistake to depend on funding external to the profession because osteopathic research is not a priority outside the profession. It is our responsibility to first make a sufficient investment and then perhaps we can realistically expect greater funding to come from the National Institutes of Health and other external sources.

The current AOA strategic plan for research calls on every member and college to financially support osteopathic research. ${ }^{9}$ The AOA can show leadership by restoring the full $\$ 50$ per membership fee investment into the Osteopathic Research and Development Fund. Anything less will be inadequate for the task. (doi:10.7556/jaoa.2014.019)

\section{Donald R. Noll, DO}

Professor of Medicine, New Jersey Institute for Successful Aging, Rowan University School of Osteopathic Medicine, Stratford

\section{References}

1. Juhl JH. Leg length discrepancy and osteoarthritic knee pain in the elderly [letter] J Am Osteopath Assoc. 2014;114(2):79-80. doi:10.7556/jaoa.2014.018.

2. Noll DR. Leg length discrepancy and osteoarthritic knee pain in the elderly: an observational study. J Am Osteopath Assoc. 2013;113(9):670-678. doi:10.7556/jaoa .2013 .033 .

3. Gurney B. Leg length discrepancy [review]. Gait Posture. 2002;15(2):195-206.

4. Sabharwal S, Kumar A. Methods for assessing leg length discrepancy [review]. Clin Orthop Relat Res. 2008;466(12):2910-2922. doi:10.1007/s11999-008-0524-9.

5. Hanada E, Kirby RL, Mitchell M, Swuste JM. Measuring leg-length discrepancy by the "iliac crest palpation and book correction" method: reliability and validity. Arch Phys Med Rehabil. 2001;82(7):938-942.

6. Juhl JH, Ippolito Cremin TM, Russell G. Prevalence of frontal plane pelvic postural asymmetry-part 1. J Am Osteopath Assoc. 2004;104(10):411-421.

7. Willman MK. Radiographic technical aspects of the postural study. J Am Osteopath Assoc. 1977;76(10):739-744.

8. Harvey WF, Yang M, Cooke TD, et al. Association of leg-length inequality with knee osteoarthritis: a cohort study. Ann Intern Med. 2010;152(5):287-295. doi:10.7326/0003-4819 -152-5-201003020-00006.

9. Degenhardt BF, Standley PR. 2013-2022 strategic plan for research: a role for everyone in promoting research in the osteopathic medical profession. J Am Osteopath Assoc. 2013;113(9):654-659.

10. 2012 Osteopathic Medical Profession Report. Chicago, IL: American Osteopathic Association; 2013. http://www.osteopathic.org/inside-aoa /about/aoa-annual-statistics/Documents/2012 -OMP-report.pdf. Accessed December 30, 2013.

11. Commission on Osteopathic College Accreditation. Colleges of osteopathic medicine. American Osteopathic Association website. http://www.osteopathic.org/inside-aoa laccreditation/predoctoral\%20accreditation /Documents/current-list-of-colleges-of -osteopathic-medicine.pdf. Updated November 18, 2013. Accessed December 30, 2013.

(๑) 2014 American Osteopathic Association 